International Journal of Pure and Applied Mathematics

Volume 105 No. 3 2015, 393-398

ISSN: 1311-8080 (printed version); ISSN: 1314-3395 (on-line version)

url: http://www.ijpam.eu

doi: http://dx.doi.org/10.12732/ijpam.v105i3.7

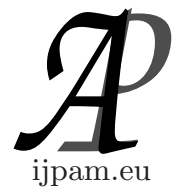

\title{
ANALYTICAL METHOD FOR SOLVING FUZZY ABEL INTEGRAL EQUATION
}

\author{
K. Balasubramanian ${ }^{1 \S}$, S. Raja Balachandar ${ }^{2}$, S.G. Venkatesh ${ }^{3}$ \\ $1,2,3$ Department of Mathematics \\ School of Humanities and Sciences \\ SASTRA University \\ Thanjavur, INDIA
}

\begin{abstract}
In this paper, we study an approximation method for solving Fuzzy Abel integral equation in the most general form. The proposed approach is based on homotopy perturbation method. The proposed method is discussed in detail and the efficiency of the method is illustrated through some numerical examples.
\end{abstract}

AMS Subject Classification: 34AXX, 34K07

Key Words: fuzzy number, fuzzy pair, fuzzy function, fuzzy Abel integral equation, homotopy perturbation method

\section{Introduction}

Abel integral equation is to describe a sliding point mass in a vertical plane on a unknown curve under gravitational force, see [12]. The point mass starts its motion without initial velocity from a point which has a vertical distance $\mathrm{x}$ from the lowest point of the curve. This equation is also occur in many branches of scientific fields, the details of the applications are presented in [11]. Recently Mirzaee (see [9]) applied homotopy analysis method for solving Fuzzy Abel integral equation. In this paper, we develop a new analytical method for solving Fuzzy Abel integral equation based on homotopy perturbation method.

Received: August 9, 2015

(c) 2015 Academic Publications, Ltd.

$\S_{\text {Correspondence author }}$ url: www.acadpubl.eu 
The concept of integration of fuzzy functions was first introduced by Dubois in [3] and later it was developed by others, see $[5,7,10]$. The fuzzy integral equation and its applications are studied in $[8,1,2]$. Basic definitions and results of fuzzy calculus which are required for this study such as fuzzy set, parametric form, metric, function, continuity, integration, etc are available in $[1,9]$.

The homotopy perturbation method (HPM) is a very powerful tool for solving various linear and nonlinear differential equations of scientific and engineering problems. Hence we implement the HPM for solving fuzzy differential equations. The HPM was first developed by He [6]. Many researchers have also investigated the solution of fuzzy differential equations using $\operatorname{HPM}[1,2,4]$. HPM has been applied to fuzzy Fredholm and Volterra integral equations by Allahviranloo et al. [1, 2].

The rest of the paper is organized as follows. In Section 2, the Fuzzy Abel integral equation and the proposed method are discussed. The numerical examples and conclusion are given in Sections 3 and 4 respectively.

\section{Homotopy Perturbation Method for Fuzzy Abel Integral Equations}

The Abel integral equation is

$$
f(x)=\int_{a}^{x} \frac{u(t)}{(x-t)^{\alpha}} d t ; \quad a \leq x \leq b,
$$

where $\alpha$ is a known constant such that $0<\alpha<1, f(x)$ is a predetermined data function and $u(x)$ is unknown function that will be determined. The expression $(x-t)^{-\alpha}$ is called the kernel of Abel integral equation, or simply Abel kernel, that is singular as $t \rightarrow x$. If $f(x)$ is a fuzzy function, these equations may only possess fuzzy solutions. Introducing the parametric forms of $f(x)$ and $u(x)$, we have the parametric form of Fuzzy Abel integral equation as follows

$$
(\underline{f}(x, r), \bar{f}(x, r))=\left(\int_{a}^{x} \frac{\underline{u}(t, r)}{(x-t)^{\alpha}} d t, \int_{a}^{x} \frac{\bar{u}(t, r)}{(x-t)^{\alpha}} d t\right) \text {, }
$$

where $0 \leq r \leq 1$ and $\alpha$ is a known constant, $\bar{f}(x)=(\underline{f}(x, r), \bar{f}(x, r))$ is a predetermined data function and $\bar{u}(x)=(\underline{u}(x, r), \bar{u}(x, r))$ is the solution that will be determined. (2) is not written in canonical form of HPM, necessary for calculating the decomposition solution series. Hence we rewrite (2) as 


$$
\begin{aligned}
& \underline{f}(x, r)=\int_{a}^{x} \frac{\underline{u}(x, r)}{\sqrt{x-t}} d t+\int_{a}^{x} \frac{\underline{u}(t, r)-\underline{u}(x, r)}{\sqrt{x-t}} d t, \\
& \underline{f}(x, r)=2 \sqrt{x-a} \underline{u}(x, r)+\int_{a}^{x} \frac{\underline{u}(t, r)-\underline{u}(x, r)}{\sqrt{x-t}} d t, \\
& \underline{u}(x, r)=\frac{\underline{f}(x, r)}{2 \sqrt{x-a}}-\frac{1}{2 \sqrt{x-a}} \int_{a}^{x} \frac{\underline{u}(t, r)-\underline{u}(x, r)}{\sqrt{x-t}} d t .
\end{aligned}
$$

Similarly

$$
\bar{u}(x, r)=\frac{\bar{f}(x, r)}{2 \sqrt{x-a}}-\frac{1}{2 \sqrt{x-a}} \int_{a}^{x} \frac{\bar{u}(t, r)-\bar{u}(x, r)}{\sqrt{x-t}} d t
$$

Let

$$
L(\underline{u}(x, r))=\underline{u}(x, r)+\frac{f(x, r)}{2 \sqrt{x-a}}-\frac{1}{2 \sqrt{x-a}} \int_{a}^{x} \frac{\underline{u}(t, r)-\underline{u}(x, r)}{\sqrt{x-t}} d t=0,
$$

where $L$ is an integral operator and we define the convex homotopy by

$$
H(\underline{u}, p)=(1-p) F(\underline{u})+p L(\underline{u}),
$$

where $F(\underline{u})=\underline{u}(x, r)-\frac{f(x, r)}{2 \overline{x-a}}$ and $p \in[0,1]$ is a embedding parameter. The initial approximate solution is taken to be $\frac{f(x, r)}{2 \frac{7}{x-a}}$ i.e. $\underline{u_{0}}(x, r)=\frac{f(x, r)}{2} \frac{\overline{x-a}}{\text {. Accord- }}$ ing to HPM, we can assume that the solution of (4) can be express as a series in $p$

$$
\begin{gathered}
\underline{u}(x, r)=\underline{u_{0}}(x, r)+p \underline{u_{1}}(x, r)+p^{2} \underline{u_{2}}(x, r)+\ldots \\
\underline{u}(x, r)=\sum_{0}^{\infty} p^{n} \underline{u_{n}}(x, r) .
\end{gathered}
$$

Similarly

$$
\bar{u}(x, r)=\sum_{0}^{\infty} p^{n} \overline{u_{n}}(x, r) .
$$

Substituting (8) in (4) and equate the coefficient of $\mathrm{p}$ with the same power, one get

$$
\begin{aligned}
& \underline{u_{0}}(x, r)=\frac{\underline{f}(x, r)}{2 \sqrt{x-a}}, \\
& \underline{u_{1}}(x, r)=\frac{-1}{2 \sqrt{x-a}} \int_{a}^{x} \frac{\underline{u_{0}}(t, r)-\underline{u_{0}}(x, r)}{\sqrt{x-t}} d t
\end{aligned}
$$




$$
\begin{array}{r}
=\underline{u_{0}}(x, r)-\frac{1}{2 \sqrt{x-a}} \int_{a}^{x} \frac{\frac{u_{0}}{\sqrt{x-t}}(t, r)}{\sqrt{x-a}} d t, \\
\underline{u_{2}}(x, r)=\underline{u_{1}}(x, r)-\frac{1}{2 \sqrt{x-a}} \int_{a}^{x} \frac{u_{1}(t, r)}{\sqrt{x-t}} d t,
\end{array}
$$

In general

$$
\underline{u_{m}}(x, r)=\underline{u_{m-1}}(x, r)-\frac{1}{2 \sqrt{x-a}} \int_{a}^{x} \frac{u_{m-1}(t, r)}{\sqrt{x-t}} d t ; \quad m \geq 1 .
$$

Similarly

$$
\overline{u_{m}}(x, r)=\overline{u_{m-1}}(x, r)-\frac{1}{2 \sqrt{x-a}} \int_{a}^{x} \frac{\overline{u_{m-1}}(t, r)}{\sqrt{x-t}} d t ; \quad m \geq 1,
$$

where $\overline{u_{0}}(x, r)=\frac{\bar{f}(x, r)}{2 \overline{x-a}}$.

Equations (8) and (9) converges to the analytic solution of (4) and (5) when $p \rightarrow 1$.

\section{Illustrated Examples}

Example 3.1. Consider the following Fuzzy Abel integral equation

$$
[r, 2-r] x=\int_{0}^{x} \frac{[\underline{u}(r, t), \bar{u}(r, t)] d t}{\sqrt{x-t}} .
$$

From (4), we get

$$
\underline{u}(x, r)=\frac{r \sqrt{x}}{2}-\frac{1}{2 \sqrt{x}} \int_{0}^{x} \frac{\underline{u}(t, r)-\underline{u}(x, r)}{\sqrt{x-t}} d t
$$

By substituting (8)into (12), we have the following results

$$
\begin{aligned}
& p^{0}: \quad \underline{u_{0}}(x, r)=f(x, r)=\frac{r \sqrt{x}}{2}, \\
& p^{1}: \quad \underline{u_{1}}(x, r)=\frac{r \sqrt{x}}{2}\left(1-\frac{\pi}{4}\right), \\
& p^{2}: \quad \underline{u_{2}}(x, r)=\frac{r \sqrt{x}}{2}\left(1-\frac{\pi}{4}\right)^{2}, \ldots
\end{aligned}
$$


Substituting the above expressions in (8), we get the analytic solution as

$$
\begin{aligned}
\underline{u}(x, r)=u_{0}+u_{1}+u_{2}+ & \cdots \\
& =\frac{r \sqrt{x}}{2}\left[1+\left(1-\frac{\pi}{4}\right)+\left(1-\frac{\pi}{4}\right)^{2}+\cdots\right]=\frac{2 r \sqrt{x}}{\pi},
\end{aligned}
$$

and $\bar{u}(x, r)=(2-r) \frac{2^{\sqrt{ }} \bar{x}}{\pi}$.

The exact solution is

$$
u(x, r)=\left(\frac{2 r \sqrt{x}}{\pi},(2-r) \frac{2 \sqrt{x}}{\pi}\right) .
$$

Example 3.2. Consider the following equation

$$
\left(\frac{4}{3} r x^{(3 / 2)}, \frac{4}{3}(2-r) x^{(3 / 2)}\right)=\int_{0}^{x} \frac{[\underline{u}(r, t), \bar{u}(r, t)]}{\sqrt{x-t}} d t,
$$

where $0 \leq r \leq 1$.

The exact solution in this case is given by

$$
(\underline{u}(x, r), \bar{u}(x, r))=(r x,(2-r) x) .
$$

Example 3.3. Consider the equation

$$
\left(\frac{5}{16}\left(r^{2}+r\right) \pi x^{3}, \frac{5}{16}\left(4-r^{3}-r\right) \pi x^{3}\right)=\int_{0}^{x} \frac{\underline{[u}(r, t), \bar{u}(r, t)]}{\sqrt{x-t}} d t .
$$

The exact solution is

$$
(\underline{u}(x, r), \bar{u}(x, r))=\left(\left(r^{2}+r\right) x^{5 / 2},\left(4-r^{3}-r\right) x^{5 / 2}\right) .
$$

\section{Conclusion}

In this paper, the Fuzzy Abel integral equations were converted into two crisp Abel integral equations based on the proposed method. Then, we applied homotopy perturbation method to obtain the unique solution of Fuzzy Abel integral equations. It was shown that this new technique is easy to implement and also be extended to solve the other type of fuzzy integral equations with some modifications. 


\section{References}

[1] T. Allahviranloo, S. Hashemzehi, The Homotopy Perturbation Method for Fuzzy Fredholm Integral Equations, Journal of Applied Mathematics, Islamic Azad University of Lahijan, 5,(2008), 1-12.

[2] T. Allahviranloo, M.Khezerloo, M. Ghanbari, S.Khezerloo, The Homotopy Perturbation Method for Fuzzy Volterra Integral Equations, International journal of computational cognition, 8, (2010), 31-37.

[3] D. Dubois, H. Prade, Towards fuzzy differential calculus, Fuzzy Sets Syst., 8,(1982), 1-7.

[4] M. Ghanbari , Numerical solution of fuzzy initial value problems under generalized differentiability by HPM, Int. J. Industrial Mathematics, 1,(2009), 19-39.

[5] R. Goetschel, W. Voxman, Elementary fuzzy calculus, Fuzzy Sets Syst., 18, (1986), 31-43.

[6] J. H. He, Homotopy perturbation technique, Computer Methods in Applied Mechanics and Engineering, 178,(1999), 257 - 262.

[7] O. Kaleva, Fuzzy differential equations, Fuzzy Sets Syst. 24,(1987), 301317.

[8] F. Mirzaee, M. Paripour, M. Komak Yari, Application of triangular and delta basis functions to solve linear Fredholm fuzzy integral equation of the second kind, Arab. J. Sci. Eng., 39, (2014), 3969-3978.

[9] F. Mirzaee, M. Komak Yari, M. Paripour, Solving linear and nonlinear Abel fuzzy integral equations by homotopy analysis method, Journal of Taibah University for Science, 9(1), (2015), 104-115.

[10] S. Nanda, On integration of fuzzy mappings, Fuzzy Sets Syst., 32,(1989), 95-101.

[11] V. Singh, R. Pandey, O. Singh, New stable numerical solutions of singular integral equations of Abel type by using normalized Bernstein polynomials, Appl. Math. Sci., 3,(2009), 241-255.

[12] A.M.Wazwaz, A first Course in Integral Equations, World Scientific, New Jersey, 1997. 